УДК 517.5

\author{
O. O. Novikov, O. G. Rovenska
}

\title{
APPROXIMATION OF PERIODIC ANALYTIC FUNCTIONS BY FÉJER SUMS
}

O. O. Novikov, O. G. Rovenska. Approximation of periodic analytic functions by Fejér sums, Mat. Stud. 47 (2017), 196-201.

For upper bounds of the deviations of Fejér sums taken over classes of periodic functions that admit analytic extensions to a fixed strip of the complex plane, we obtain asymptotic equalities. In certain cases, these equalities give a solution of the corresponding KolmogorovNikolsky problem.

1. Introduction. Let $C_{\beta, \infty}^{q}$ (see, e.g., [14]) be classes of continuous $2 \pi$-periodic functions given by the convolutions

$$
f(x)=A_{0}+\frac{1}{\pi} \int_{-\pi}^{\pi} f_{\beta}^{q}(x+t) P_{\beta}^{q}(t) d t .
$$

Here,

$$
P_{\beta}^{q}(t)=\sum_{k=1}^{\infty} q^{k} \cos \left(k t+\frac{\beta \pi}{2}\right), q \in(0 ; 1), \beta \in \mathbb{R}^{1},
$$

is the well-known Poisson kernel, the function $f_{\beta}^{q}(\cdot) \in L$ satisfies the condition

$$
\left\|f_{\beta}^{q}(\cdot)\right\|_{\infty}=\operatorname{ess} \sup \left|f_{\beta}^{q}(\cdot)\right| \leq 1 \text {. }
$$

In this case, the sets $C_{\beta, \infty}^{q}$ consist of $2 \pi$-periodic functions that admit extension to functions $f(z)=f(x+i y)$ analytic in the strip $|y|<\ln \left(\frac{1}{q}\right)$ (see, e.g., [14]); these functions are called the Poisson integrals.

Further, let $f(x)$ be a $2 \pi$-periodic summable function $(f \in L)$,

$$
S[f]=\frac{a_{0}}{2}+\sum_{k=1}^{\infty}\left(a_{k} \cos k x+b_{k} \sin k x\right)
$$

be its trigonometric Fourier series, $a_{k}=a_{k}(f)$ and $b_{k}=b_{k}(f), k=0,1, \ldots$, be its trigonometric Fourier coefficients.

2010 Mathematics Subject Classification: $42 \mathrm{~A} 10$.

Keywords: asymptotic equality; analytic functions; Fejér sums; de la Vallee Poussin sums; Poisson kernel. doi:10.15330/ms.47.2.196-201

(C) O. O. Novikov, O. G. Rovenska, 2017 
Denote by $\Lambda=\left\|\lambda_{k}^{(n)}\right\|, k, n=0,1, \ldots$ an arbitrary triangular matrix of numbers by using which we associate every function $f \in L$ with a sequence of trigonometric polynomials $U_{n}(f ; x ; \Lambda)$ of the form

$$
U_{n}(f ; x ; \Lambda)=\frac{a_{0}}{2}+\sum_{k=1}^{n-1} \lambda_{k}^{(n)}\left(a_{k} \cos k x+b_{k} \sin k x\right) .
$$

Thus, any triangular matrix $\Lambda$ gives a method for the construction of polynomials $U_{n}(f ; x ; \Lambda)$ or, in other words, specific sequence of polynomial operators $U_{n}(f ; \Lambda)$ defined on the set $L$. In this case, one also says that the matrix $\Lambda$ defines a specific method $(\Lambda$-method) for summing Fourier series. It is clear that the operators $U_{n}(f ; \Lambda)$ are linear. For this reason, $\Lambda$-methods are called linear summation methods for Fourier series [13].

For arbitrary natural $p<n$ the polynomials that are given by relationship

$$
\lambda_{k}^{(n)}= \begin{cases}1, & 1 \leq k \leq n-p \\ 1-\frac{k-n+p}{p}, & n-p+1 \leq k \leq n-1\end{cases}
$$

are called de la Vallee Poussin sums. In this case, the polynomials $U_{n}(f ; x ; \Lambda)$ are denoted by $V_{n, p}(f, x)$ and have the form

$$
V_{n, p}(f ; x)=\frac{1}{p} \sum_{k=n-p}^{n-1} S_{k}(f ; x)
$$

where $S_{k}(f ; x), k=0,1, \ldots$ are the partial Fourier sums of order $k$ of the function $f(x)$. If $p=1$, then $V_{n, p}(f ; x)=S_{n-1}(f ; x)$. For $p=n$ sums of this type are called the Fejér sums:

$$
\sigma_{n}(f ; x)=V_{n, n}(f ; x)=\frac{1}{n} \sum_{k=0}^{n-1} S_{k}(f ; x) .
$$

We say that the Kolmogorov-Nikolsky problem is solved for given method $U_{n}(f ; \Lambda)$ on a class $\mathfrak{N}$ if a function $\varphi(n)=\varphi(n ; \Lambda ; \mathfrak{N})$ such that

$$
\mathcal{E}\left(\mathfrak{N} ; U_{n}\right)=\sup _{f \in \mathfrak{N}}\left\|f(x)-U_{n}(f ; x ; \Lambda)\right\|_{C}=\varphi(n)+o(\varphi(n)) \quad \text { as } n \rightarrow \infty
$$

is determined in explicit form [13].

De la Vallee-Poussin sums and their special cases (Fourier sums and Fejér sums) have been extensively studied for many decades by many prominent experts in the theory of functions. In 1946, Nikolsky ([2]) considered the quantity

$$
\mathcal{E}\left(C_{\beta, \infty}^{q} ; S_{n}\right)=\sup _{f \in C_{\beta, \infty}^{q}}\left\|f(x)-S_{n}(f ; x)\right\|_{C}
$$

where $S_{n}(f ; x)$ is the $n$-th partial sum of the trigonometric Fourier series of the function $f(x)$, and established the asymptotic equality (as $n \rightarrow \infty)$

$$
\mathcal{E}\left(C_{\beta, \infty}^{q} ; S_{n}\right)=\frac{8 q^{n}}{\pi^{2}} K(q)+O(1) \frac{q^{n}}{n}, \quad K(q)=\int_{0}^{\frac{\pi}{2}} \frac{d u}{\sqrt{1-q^{2} \sin ^{2} u}},
$$


where $K(q)$ is the total elliptic integral of the first kind, $O(1)$ is a quantity uniformly bounded with respect to $n$. In 1980, Stechkin ([12]) proposed another proof of this result, which allowed to refine the remainder equality

$$
\mathcal{E}\left(C_{\beta, \infty}^{q} ; S_{n}\right)=\frac{8 q^{n}}{\pi^{2}} K(q)+O(1) \frac{q^{n}}{n(1-q)},
$$

where $O(1)$ is a quantity uniformly bounded with respect to $n, q$.

It follows from these equalities that, on the classes of analytic functions, the trigonometric Fourier sums provide an approximation which coincides in order with the best approximation by the trigonometric polynomials of degree not greater than $n$. However, it is still interesting to make clear how do other approximating aggregates (e.g., de la Vallee-Poussin sums, Fejér sums) behave on the classes mentioned above [8]. These studies may be of interest for computational mathematics and methods of mathematical modeling.

Asymptotic (as $n \rightarrow \infty$ ) equalities for upper bounds of the deviations of de la Vallee Poussin sums for $p$ satisfying $n-p \rightarrow \infty$ on the classes $C_{\beta, \infty}^{q}$ may be found in [11] (also [8]):

$$
\begin{gathered}
\mathcal{E}\left(C_{\beta, \infty}^{q} ; V_{n, p}\right)=\sup _{f \in C_{\beta, \infty}^{q}}\left\|f(x)-V_{n, p}(f ; x)\right\|_{C}= \\
=\frac{q^{n-p+1}}{p}\left(\frac{4}{\pi^{2}} K_{q, p}+O(1)\left(\frac{q}{(n-p+1)(1-q)^{s}}\right)\right),
\end{gathered}
$$

where

$$
K_{q, p}=\int_{0}^{\pi} \frac{\sqrt{1-2 q^{p} \cos p t+q^{2 p}}}{1-2 q \cos p t+q^{2}} d t, \quad s=s(p)= \begin{cases}1, & p=1 \\ 3, & p=2,3, \ldots\end{cases}
$$

$O(1)$ is a quantity uniformly bounded with respect to $n, p, q$.

In the case of arbitrary $p=1,2, \ldots, n$ the behaviour of the constant $K_{q, p}$ could be inferred by the next identity, proved in [10]:

$$
K_{q, p}=2 \frac{1-q^{2 p}}{1-q^{2}} K\left(q^{p}\right),
$$

where $K(q)$ is the total elliptic integral of the first kind.

Some related problems were studied in $[1,3,4,5,6,7,9]$.

In the present paper we are interested in asymptotic equalities for the quantifies

$$
\mathcal{E}\left(C_{0, \infty}^{q} ; \sigma_{n}\right)=\sup _{f \in C_{0, \infty}^{q}}\left\|f(x)-\sigma_{n}(f ; x)\right\|_{C}
$$

2. Results. Our main result is in the following theorem.

Theorem. Suppose that $q \in(0 ; 2-\sqrt{3}$. Then the following relations hold as $n \rightarrow \infty$

$$
\mathcal{E}\left(C_{0, \infty}^{q}, \sigma_{n}\right)=\frac{4 q}{\pi n\left(1+q^{2}\right)}+O(1) \frac{q^{n}}{n}
$$

where $O(1)$ is a quantity uniformly bounded with respect to $n, q$. 
Proof. Let

$$
\delta_{n}(f ; x)=f(x)-\sigma_{n}(f ; x)=f(x)-\frac{1}{n} \sum_{k=0}^{n-1} S_{k}(f ; x)=\frac{1}{n} \sum_{k=0}^{n-1} \rho_{k}(f ; x),
$$

where $\rho_{k}(f ; x)=f(x)-S_{k}(f ; x)$.

For $f \in C_{0, \infty}^{q}$ quantity $\rho_{m}(f ; x)$ may be represented as follows

$$
\begin{aligned}
\rho_{m}(f ; x) & =\frac{1}{\pi} \int_{-\pi}^{\pi} f_{0}^{q}(x+t) \sum_{k=m+1}^{\infty} q^{k} \cos k t d t=\frac{1}{\pi} \int_{-\pi}^{\pi} f_{0}^{q}(x+t) \sum_{k=0}^{\infty} q^{k+m+1} \cos (k+m+1) t d t= \\
& =\frac{q^{m+1}}{\pi} \int_{-\pi}^{\pi} f_{0}^{q}(x+t)\left(\cos (m+1) t \sum_{k=0}^{\infty} q^{k} \cos k t-\sin (m+1) t \sum_{k=0}^{\infty} q^{k} \sin k t\right) d t .
\end{aligned}
$$

According to formulas

$$
\sum_{k=0}^{\infty} q^{k} \cos k t=\frac{1-q \cos t}{1-2 q \cos t+q^{2}}, \quad \sum_{k=0}^{\infty} q^{k} \sin k t=\frac{q \sin t}{1-2 q \cos t+q^{2}},
$$

we obtain

$\rho_{m}(f ; x)=\frac{q^{m+1}}{\pi} \int_{-\pi}^{\pi} f_{0}^{q}(x+t)\left\{\frac{1-q \cos t}{1-2 q \cos t+q^{2}} \cos (m+1) t-\frac{q \sin t}{1-2 q \cos t+q^{2}} \sin (m+1) t\right\} d t$.

Comparing (2) and (3), we obtain

$$
\begin{gathered}
\delta_{n}(f ; x)=\frac{1}{n} \sum_{k=0}^{n-1} \rho_{k}(f ; x)= \\
=\frac{1}{\pi n} \sum_{m=1}^{n} \int_{-\pi}^{\pi} f_{0}^{q}(x+t) q^{m}\left[\frac{(1-q \cos t) \cos m t}{1-2 q \cos t+q^{2}}-\frac{q \sin t \sin m t}{1-2 q \cos t+q^{2}}\right] d t= \\
=\frac{1}{\pi n} \int_{-\pi}^{\pi} \frac{f_{0}^{q}(x+t)}{1-2 q \cos t+q^{2}} \sum_{m=1}^{n} q^{m}[\cos m t-q \cos (m-1) t] d t .
\end{gathered}
$$

We have

$$
\begin{gathered}
\sum_{k=1}^{n} q^{k}(\cos k t-q \cos (k-1) t)=\sum_{k=1}^{n} q^{k}\left(\frac{e^{i k t}+e^{-i k t}}{2}-q \frac{e^{i(k-1) t}+e^{-i(k-1) t}}{2}\right)= \\
=\frac{1}{2} \sum_{k=1}^{n}\left\{\left[\left(q e^{i t}\right)^{k}+\left(q e^{-i t}\right)^{k}\right]-q^{2}\left[\left(q e^{i t}\right)^{k-1}+\left(q e^{-i t}\right)^{k-1}\right]\right\}= \\
=\frac{1}{1-2 q \cos t+q^{2}}\left[\left(q+q^{3}\right) \cos t-2 q^{2}-q^{n+1}\left(\cos (n+1) t-2 q \cos n t+q^{2} \cos (n-1) t\right)\right] .
\end{gathered}
$$


Taking into account the formula (4), the following integral representation holds as $n \rightarrow \infty$

$$
\delta_{n}(f ; x)=\frac{q}{\pi n} \int_{-\pi}^{\pi} \frac{f_{0}^{q}(x+t)\left[\left(1+q^{2}\right) \cos t-2 q\right]}{\left(1-2 q \cos t+q^{2}\right)^{2}} d t+O(1) \frac{q^{n}}{n},
$$

where $O(1)$ is a quantity uniformly bounded with respect to $n, q$.

The function

$$
\Gamma(t)=\frac{\left(1+q^{2}\right) \cos t-2 q}{\left(1-2 q \cos t+q^{2}\right)^{2}}
$$

for $q \in(0 ; 2-\sqrt{3}$ monotonous on interval $(0 ; \pi)$. Using the relation $(5)$, we conclude that for $f_{0}^{q}(t) \in S_{M}^{0}$,

$$
S_{M}^{0}=\left\{f \in L \vdots \quad \int_{-\pi}^{\pi} f(t) d t=0, \quad \operatorname{ess} \sup |f(t)| \leq 1, \quad t \in[-\pi ; \pi]\right\},
$$

the following representation

$$
\delta_{n}(f ; x)=\frac{q}{\pi n} \int_{-\pi}^{\pi} f_{0}^{q}(x+t)\left(\frac{\left(1+q^{2}\right) \cos t-2 q}{\left(1-2 q \cos t+q^{2}\right)^{2}}-\frac{-2 q}{\left(1+q^{2}\right)^{2}}\right) d t+O(1) \frac{q^{n}}{n}
$$

is true.

For $q \in(0 ; 2-\sqrt{3}]$ we have $\varphi(t)=\operatorname{sign}[\Gamma(t)-\Gamma(\pi / 2)] \in S_{M}^{0}$. Taking into account that $f_{0}^{q}(x)=\varphi(x)$, we obtain

$$
\begin{aligned}
\mathcal{E}\left(C_{0, \infty}^{q}, \sigma_{n}\right)= & \frac{q}{\pi n} \int_{-\pi}^{\pi} \varphi(t)\left(\frac{\left(1+q^{2}\right) \cos t-2 q}{\left(1-2 q \cos t+q^{2}\right)^{2}}+\frac{2 q}{\left(1+q^{2}\right)^{2}}\right) d t+O(1) \frac{q^{n}}{(1-q)^{3} n}= \\
& =\frac{2 q}{\pi n} \int_{0}^{\pi}\left|\frac{\left(1+q^{2}\right) \cos t-2 q}{\left(1-2 q \cos t+q^{2}\right)^{2}}+\frac{2 q}{\left(1+q^{2}\right)^{2}}\right| d t+O(1) \frac{q^{n}}{n} .
\end{aligned}
$$

It is clear that

$$
\begin{gathered}
\int_{0}^{\pi}\left|\frac{\left(1+q^{2}\right) \cos t-2 q}{\left(1-2 q \cos t+q^{2}\right)^{2}}+\frac{2 q}{\left(1+q^{2}\right)^{2}}\right| d t= \\
=\int_{0}^{\pi / 2} \frac{\left(1+q^{2}\right) \cos t-2 q}{\left(1-2 q \cos t+q^{2}\right)^{2}} d t-\int_{\pi / 2}^{\pi} \frac{\left(1+q^{2}\right) \cos t-2 q}{\left(1-2 q \cos t+q^{2}\right)^{2}} d t .
\end{gathered}
$$

Making calculations, we obtain

$$
\begin{gathered}
\int \frac{\cos t}{\left(1-2 q \cos t+q^{2}\right)^{2}} d t=\frac{\left(1+q^{2}\right) \sin t}{\left(1-q^{2}\right)^{2}\left(1-2 q \cos t+q^{2}\right)}+\frac{2 q}{\left(1-q^{2}\right)^{2}} \int \frac{d t}{1-2 q \cos t+q^{2}}, \\
\int \frac{d t}{\left(1-2 q \cos t+q^{2}\right)^{2}}=\frac{2 q \sin t}{\left(1-q^{2}\right)^{2}\left(1-2 q \cos t+q^{2}\right)}+\frac{1+q^{2}}{\left(1-q^{2}\right)^{2}} \int \frac{d t}{1-2 q \cos t+q^{2}} .
\end{gathered}
$$


Making elementary transformations, we obtain

$$
\begin{gathered}
\int \frac{\left(1+q^{2}\right) \cos t-2 q}{\left(1-2 q \cos t+q^{2}\right)^{2}} d t=\frac{\sin t}{1-2 q \cos t+q^{2}}, \\
\int_{0}^{\pi / 2} \frac{\left(1+q^{2}\right) \cos t-2 q}{\left(1-2 q \cos t+q^{2}\right)^{2}} d t-\int_{\pi / 2}^{\pi} \frac{\left(1+q^{2}\right) \cos t-2 q}{\left(1-2 q \cos t+q^{2}\right)^{2}} d t=\frac{2}{1+q^{2}} .
\end{gathered}
$$

We get asymptotic formula (1) based on (6), (7).

The formula (1) provides the solving of the Kolmogorov-Nikolsky problem when performing the theorems conditions without any additional conditions.

\section{REFERENCES}

1. Velichko V.E., Novikov O.A., Rovenskaya O.G., Rukasov V.I., Approximation of analytic functions by repeated de la Vallee Poussin sums, Tr. Inst. Prikl. Mat. Mekh., 22 (2011), 33-42. (in Russian)

2. Nikolskiy S.M., Approximation of the functions by trigonometric polynomials in the mean, Izv. Acad. Nauk. SSSR, Ser. Mat., 10 (1946), №3, 207-256. (in Russian)

3. Novikov O.O., Rovenska O.G., Approximation of the periodical functions of high smoothness by the rightangled Fourier sums, Carpathian Mathematical Publications, 5 (2013), №1, 111-118. (in Ukrainian)

4. Novikov O.A., Rovenska O.G., Approximation of classes of Poisson integrals by Fejér sums, Computer Research and Modeling, 7 (2015), №4, 813-819. (in Russian)

5. Novikov O.O., Rovenska O.G., Kozachenko Yu.V., Approximation of Poisson integrals by Fejér sums, Modern problems of probability theory and mathematical analysis. Scientific conference, Vorohta, 2016, 110. (in Ukrainian)

6. Rovenska O.G., Integral presentations of deviations of right-angled linear means of Fourier series on classes $C^{m \psi}$, Bukovinsky Mat. Zh., 1 (2011), №3, 99-104. (in Ukrainian)

7. Rovenska O.O., Novikov O.O., Approximation of Poisson integrals by repeated de la Vallee Poussin sums, Nonlinear Oscillations, 13 (2010), №1, 108-111.

8. Rukasov V.I., Chaichenko, S.O., Approximation of the classes of analytical functions by de la ValleePoussin sums, Ukrainian Math. J., 54 (2002), №12, 2006-2024.

9. Rukasov V., Rovenska O., Integral presentations of deviations of de la Vallee Poussin right-angled sums, Acta Univ. Palacki. Olomuc., Fac. rer. nat., Math., 48 (2009), 129-137.

10. Savchuk V.V., Savchuk M.V., Chaichenko S.O., Approximation of analytic functions by de la Vallee Poussin sums, Mat. Stud., 34 (2010), №2, 207-219. (in Ukrainian)

11. Serdyuk A.S., Approximation of Poisson integrals by de la Vallee Poussin sums, Ukrainian Math. J., 56 (2004), №4, 122-134.

12. Stechkin S.B., Estimation of the remainder of Fourier series for the differentiable functions, Tr. Mat. Inst. Acad. Nauk SSSR., 145 (1980), 126-151. (in Russian)

13. Stepanec A.I., Classification and Approximation of Periodic Functions, Naukova Dumka, Kiev, 1987. (in Russian)

14. Stepanec A.I., Solution of the Kolmogorov-Nikol'skij problem for the Poisson integrals of continuous functions, Mat. Sb., 192 (2001), №1, 113-138. (in Russian)

Donbas State Pedagogical University, Slov'yansk, Ukraine sgpi.slav@dn.ua

Donbas State Engineering Academy, Kramatorsk, Ukraine rovenskaya.olga.math@gmail.com 